\title{
Correction to: Integrated Extremal Control and Explicit Guidance for Quadcopters
}

\author{
Evan Kawamura ${ }^{1}$. Dilmurat Azimov ${ }^{2}$
}

Published online: 5 April 2021

(C) Springer Nature B.V. 2021

Correction to: Journal of Intelligent Robotics System https://doi.org/10.1007/s10846-020-01211-2

Due to graduating and obtaining a job after graduation, the first author's affiliation has changed from the previous manuscript. Table 1 shows the previous and current affiliation of the first author.

The original article can be found online at https://doi.org/10.1007/ s10846-020-01211-2.

Evan Kawamura

evan.t.kawamura@nasa.gov

Dilmurat Azimov

azimov@hawaii.edu

1 Intelligent Systems - Autonomous Systems \& Robotics, NASA Ames Research Center, Moffett Field, Mountain View, CA, 94035, USA

2 Mechanical Engineering, Holmes Hall 302, 2540 Dole Street, Honolulu, HI, 96822, USA
Due to mixing time computations for position commands and real world clock time, figures 10, 11, 14, 18, 20, 21, 22, and 23 of Ref. [2] have the wrong times for the experimental and HITL data. Figures 1, 2, 3, 4, 5, 6, 7 and 8 of this corrigendum replace Figs. 10, 11, 14, 18, 20, 21, 22, and 23 of Ref. [2], respectively.

The PID controller finishes before the extremal controller (flight test and HITL) because the elapsed time in the DJI OSDK implementation was a counter for computing position commands instead of the real world clock time. Therefore, the PID controller has a higher average velocity of $2.5 \mathrm{~m} / \mathrm{s}$ as it ascends to $20 \mathrm{~m}$ in approximately 8 seconds, while the extremal control has an average velocity of $0.71 \mathrm{~m} / \mathrm{s}$ with an ascent time of 28 seconds (see Fig. 11 of Ref. [2]). Consequently, the PID velocity profile is significantly larger than the integrated extremal control and explicit guidance velocity. The PID velocity profile resembles a classic trapezoidal velocity profile for point to point maneuvers with max acceleration (max force), constant velocity, and then max deceleration [1, 3]. This typical approach is time optimal due to $\max$ acceleration and velocity but has discontinuous acceleration profiles [1]. Contrarily, the integrated extremal control and explicit guidance method presented here has continuous acceleration through E Guidance, which yields smoother profiles for velocity and position. Similar results occur for the waypoint guidance maneuver, but the real world clock time is 68 seconds. 
Table 1 First author affiliation update

$\begin{array}{ll}\text { Previous Current } & \end{array}$

Department

Address 1

Address 2

Email

\section{Mechanical Engineering}

University of Hawaii: Manoa

Holmes Hall 2540 Dole Street

Honolulu, HI 96822, USA

evankawa@hawaii.edu
Intelligent Systems

Autonomous Systems \& Robotics

NASA Ames Research Center

Moffett Field, Mountain View, CA 94035

evan.t.kawamura@nasa.gov

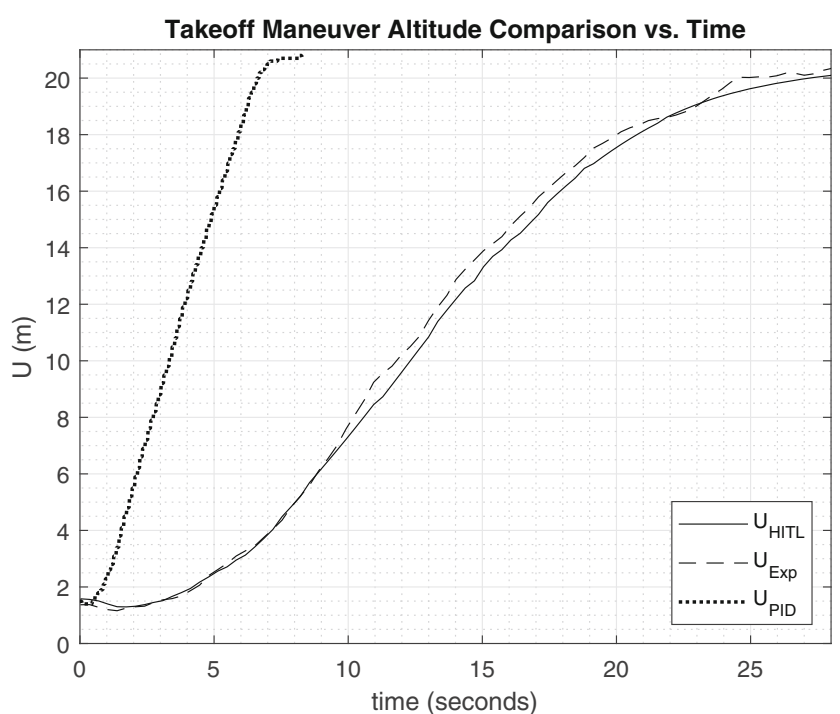

Fig. 1 Takeoff maneuver: Altitude comparison (replaces Fig. 10 of Ref. [2])

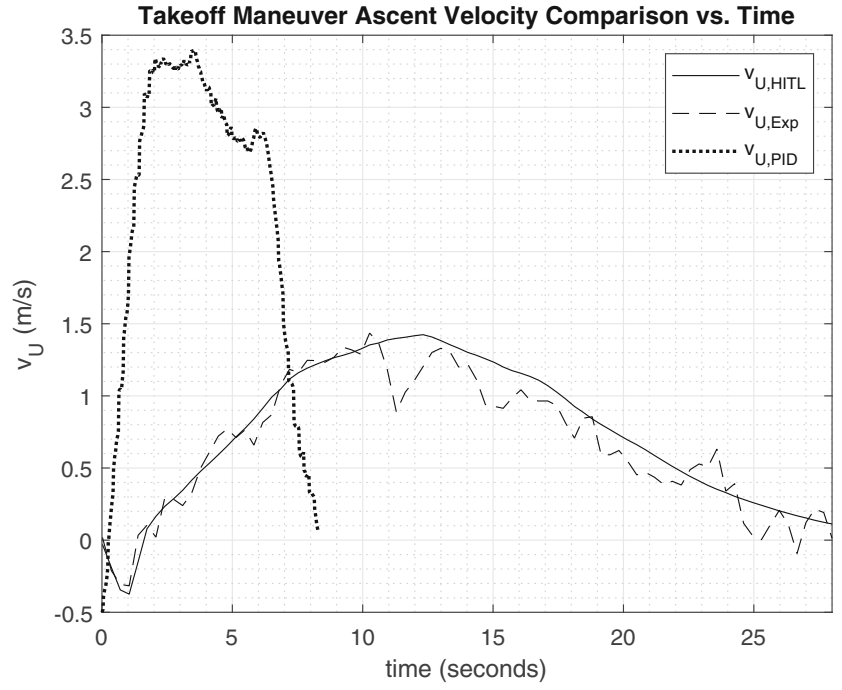

Fig. 2 Takeoff maneuver: Velocity comparison (replaces Fig. 11 of Ref. [2])

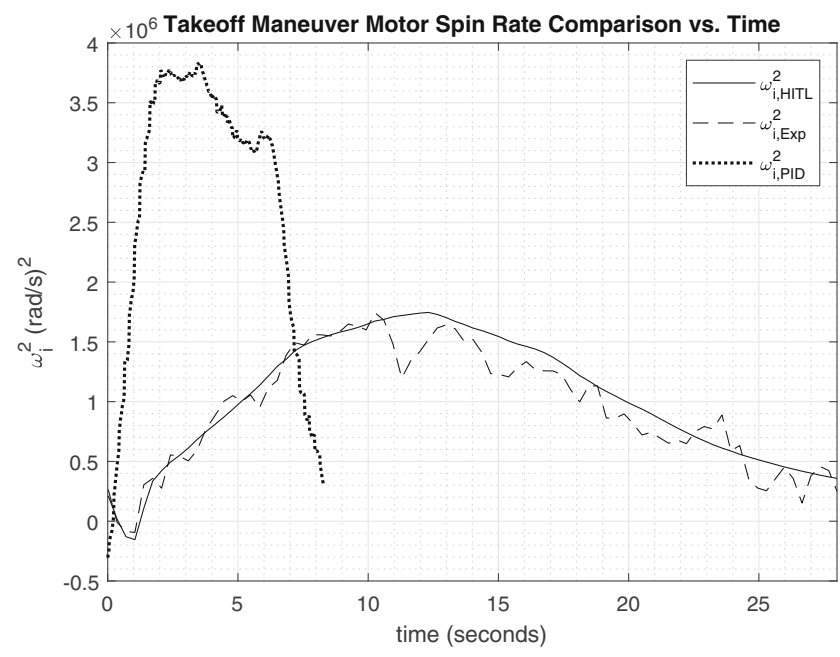

Fig. 3 Takeoff maneuver motor spin rate comparison (replaces Fig. 14 of Ref. [2]) 
Fig. 4 Waypoint guidance: Experimental, HITL, \& PID velocity comparison vs. Time (replaces Fig. 18 of Ref. [2])
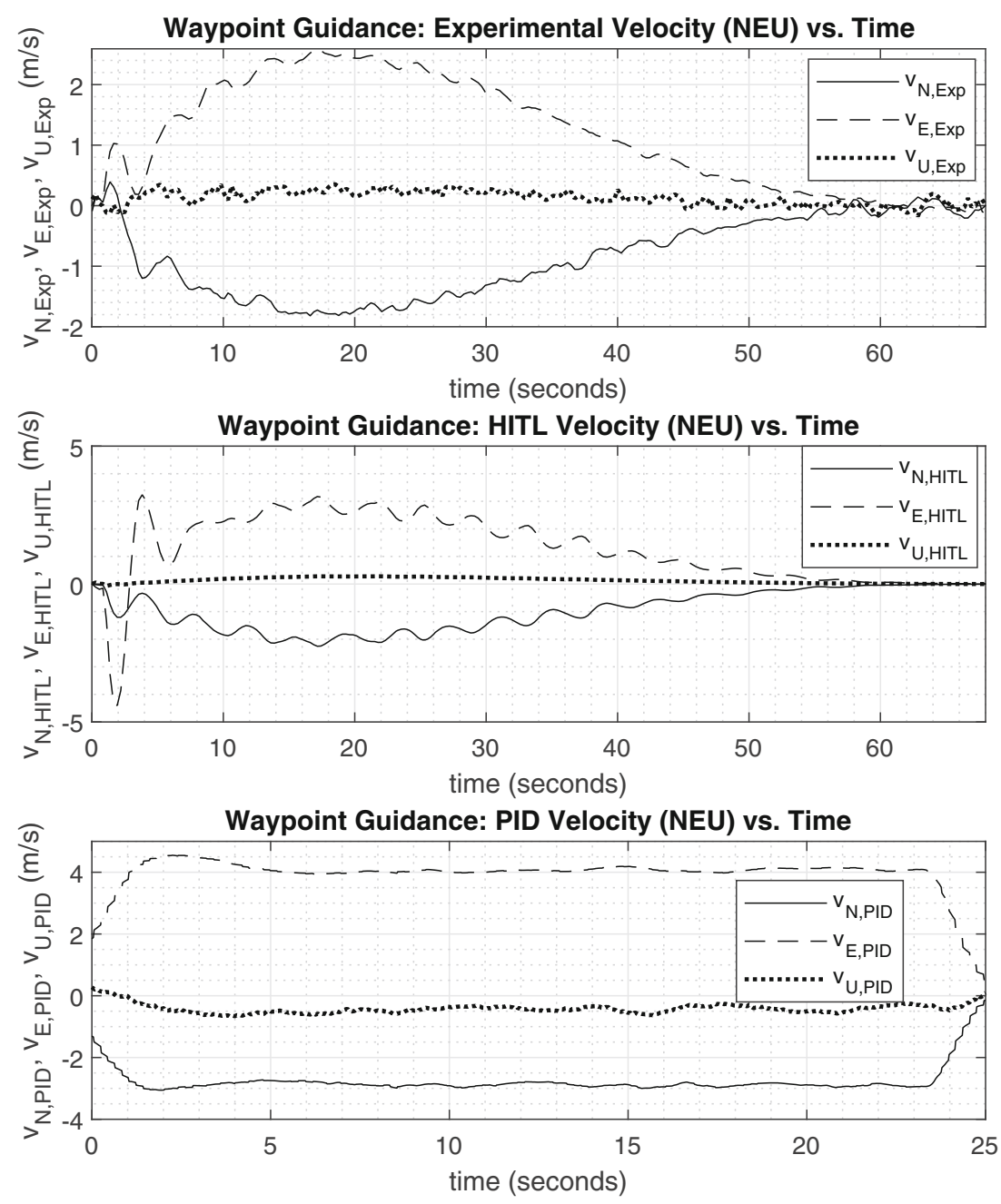
Fig. 5 Waypoint guidance: Experimental, HITL, \& PID position comparison vs. Time (replaces Fig. 20 of Ref. [2])
Waypoint Guidance: Experimental Positions (NEU) vs. Time

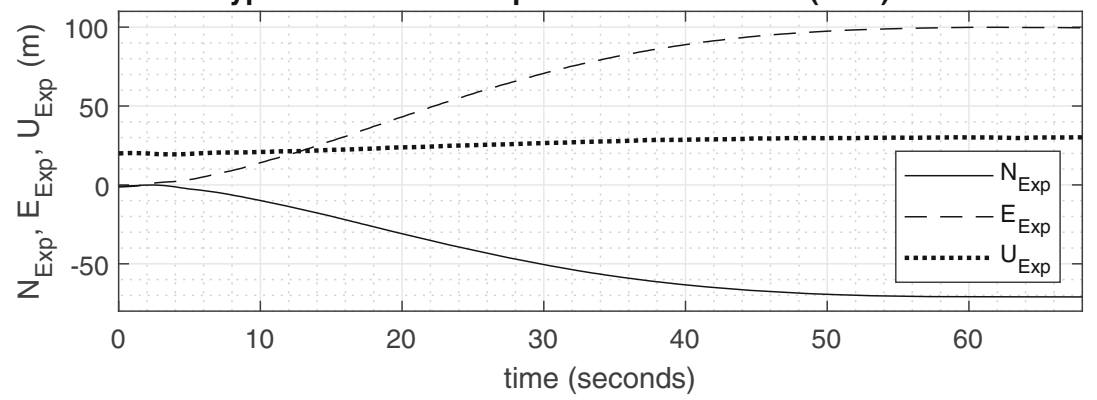

Waypoint Guidance: HITL Positions (NEU) vs. Time

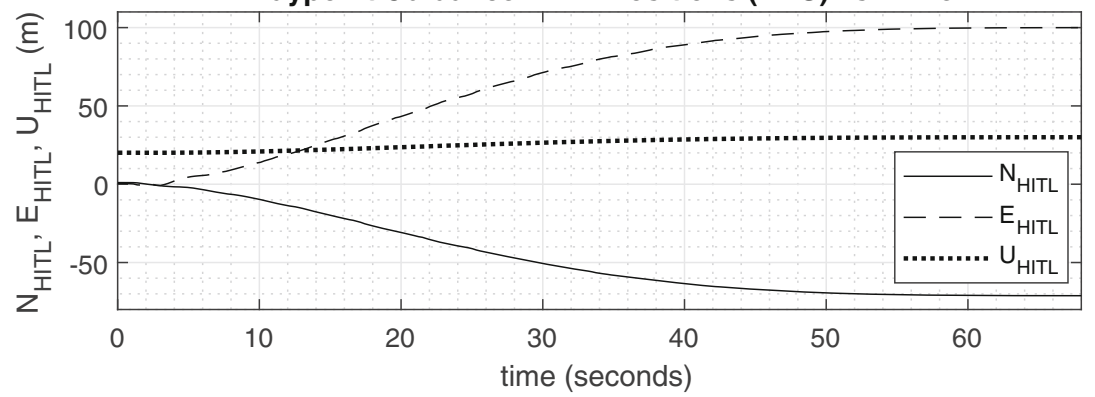

Waypoint Guidance: PID Positions (NEU) vs. Time

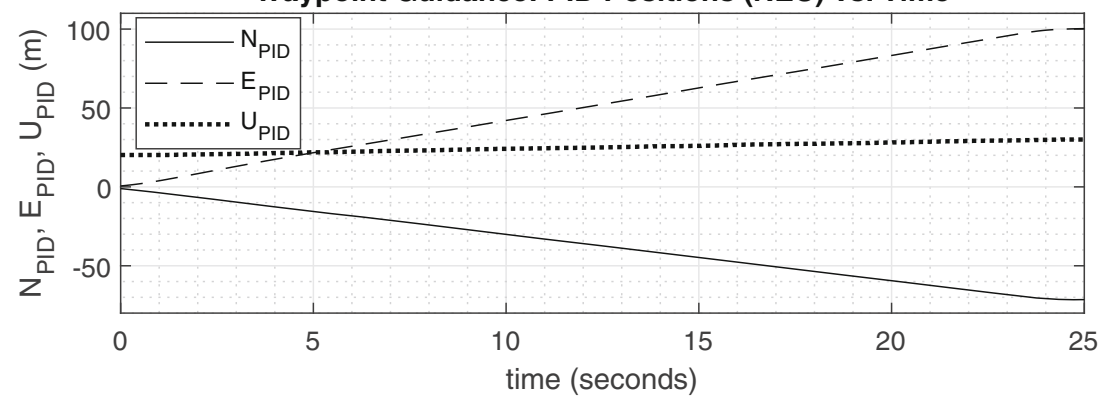


Fig. 6 Waypoint guidance experimental, HITL, \& PID motor spin rate comparison vs. Time (replaces Fig. 21 of Ref.

[2])
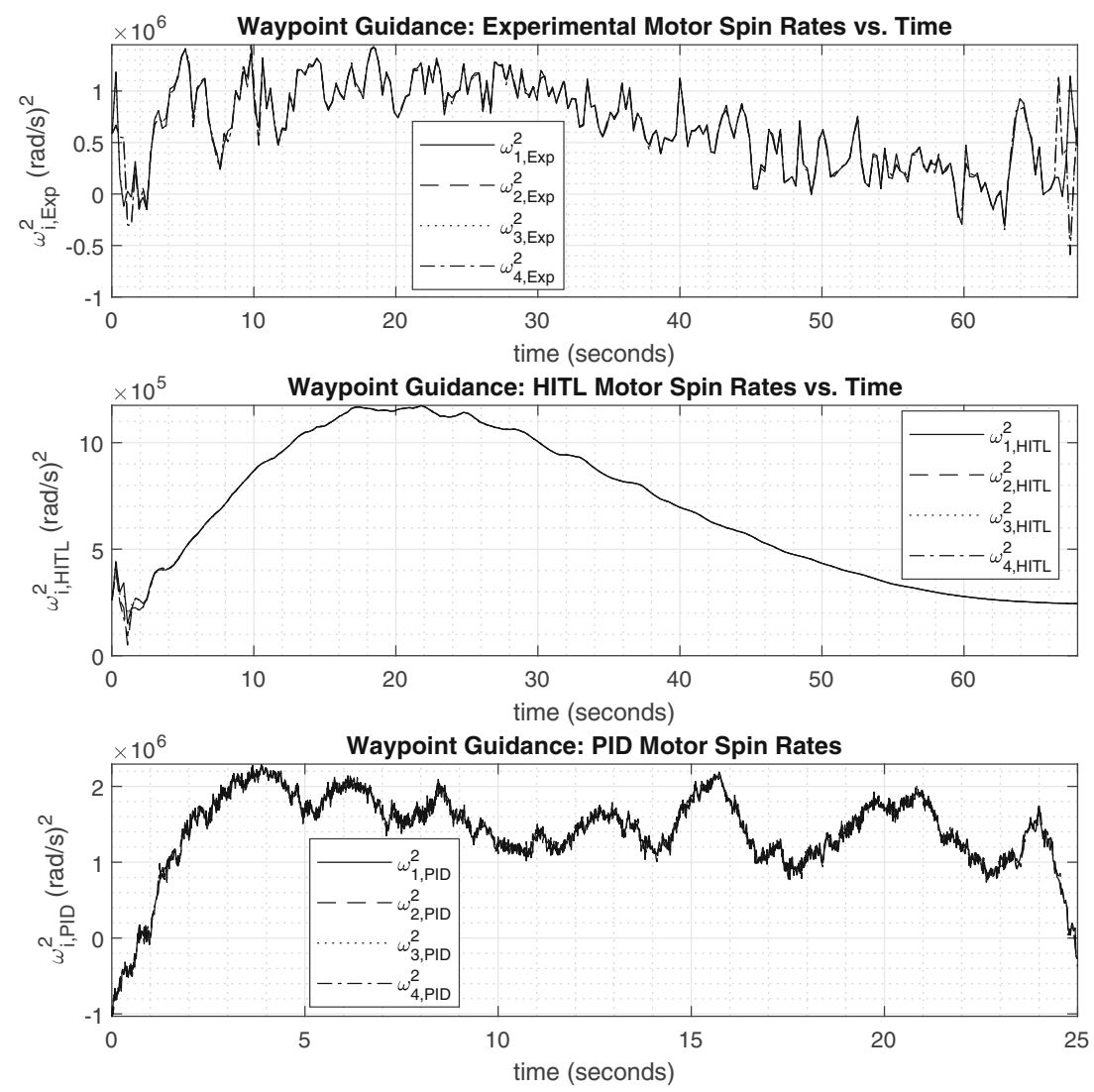
Fig. 7 Waypoint guidance: E guidance \& PID euler angles comparison vs. Time (replaces Fig. 22 of Ref. [2])
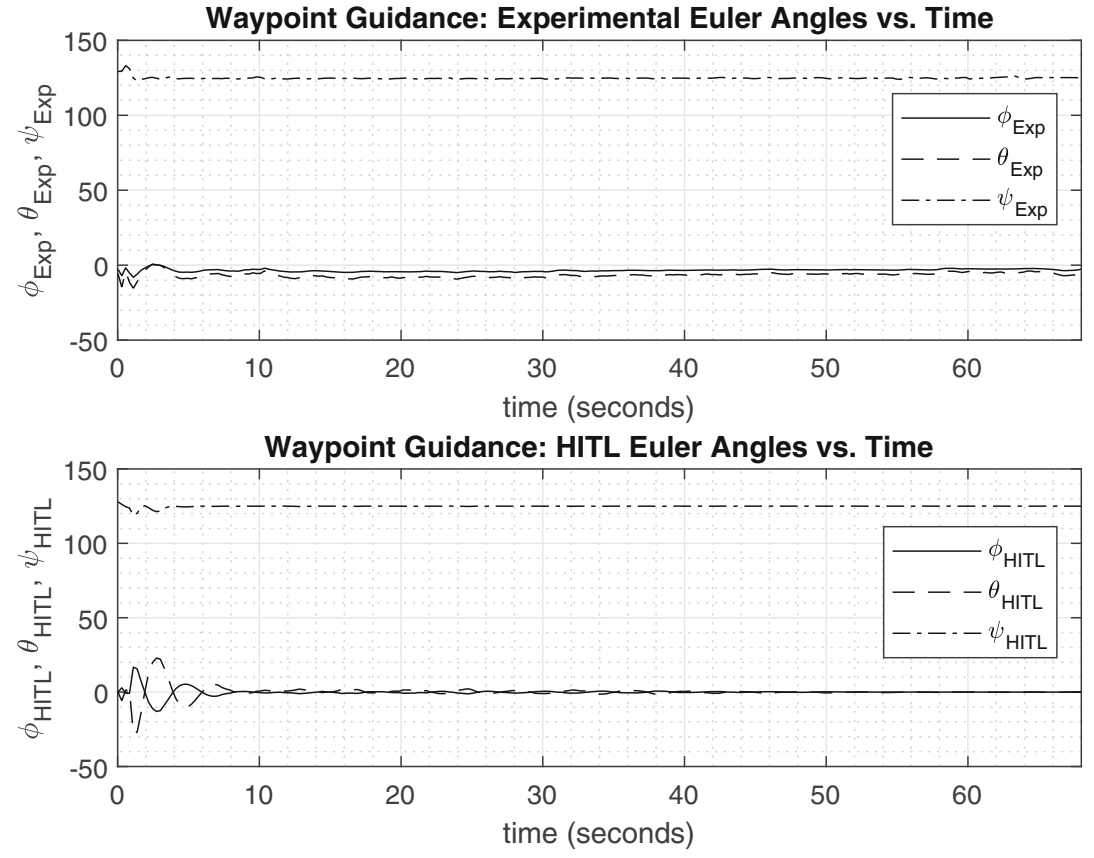

Waypoint Guidance: PID Euler Angles vs. Time

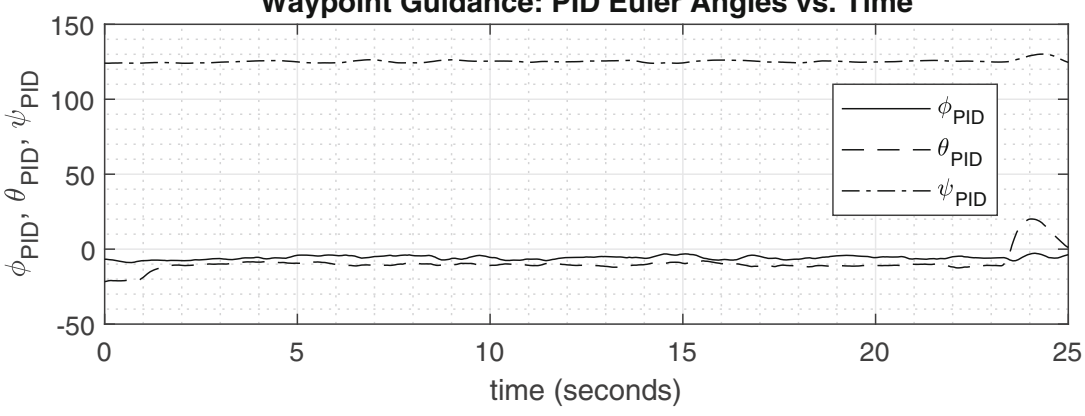


Fig. 8 Waypoint guidance: E guidance \& PID quaternion comparison vs. Time (replaces Fig. 23 of Ref. [2])
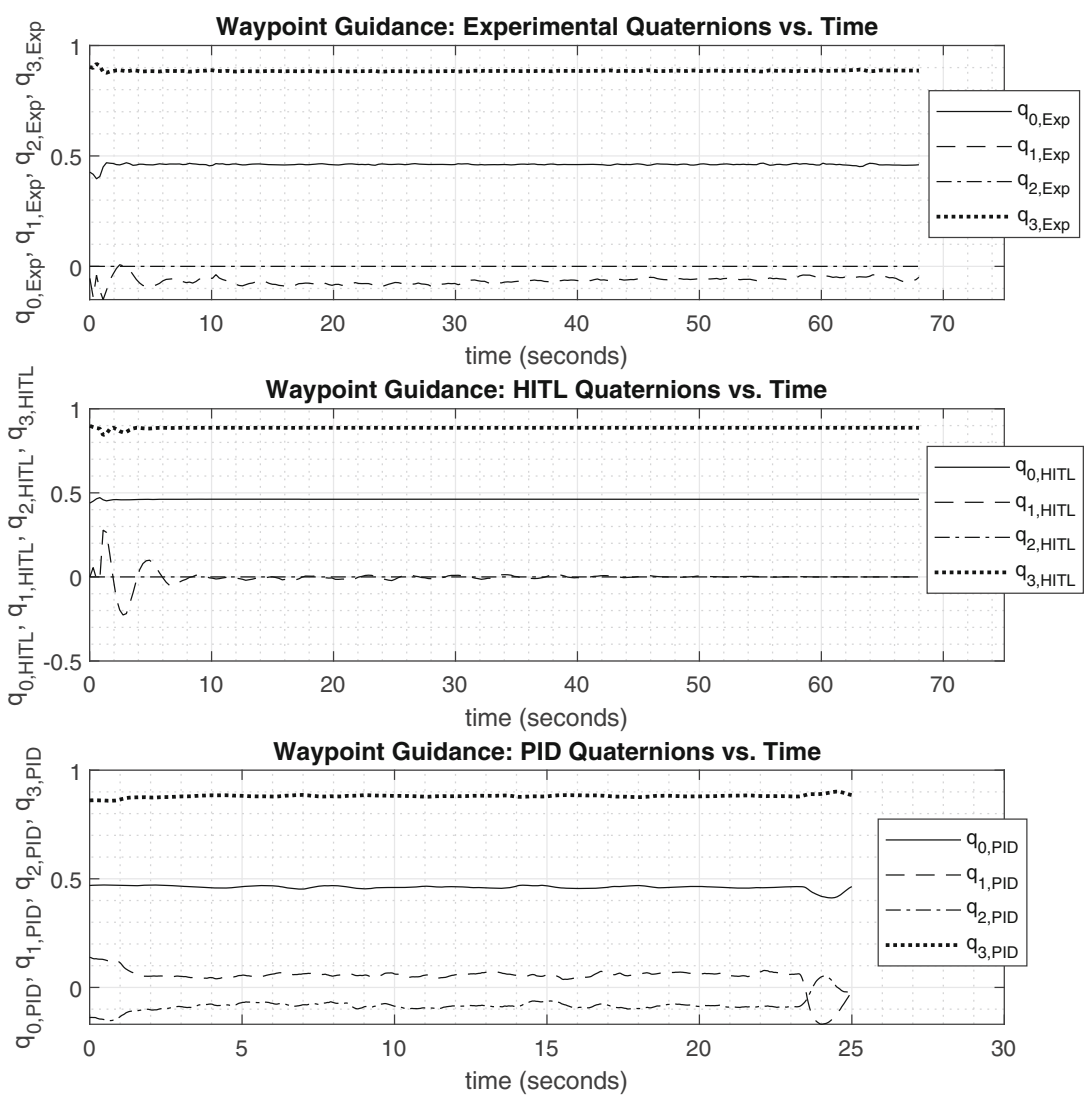

Acknowledgements The authors would like to thank Dr. Peter Berkelman for pointing out the discrepancy with the average velocity for the takeoff maneuver, which led to further investigation to find the error in computing the real world time for the experimental and HITL data.

\section{References}

1. Haschke, R., Weitnauer, E., Ritter, H.: On-line planning of timeoptimal, jerk-limited trajectories. In: 2008 IEEE/RSJ International
Conference on Intelligent Robots and Systems, pp. 3248-3253. IEEE (2008)

2. Kawamura, E., Azimov, D.: Integrated extremal control and explicit guidance for quadcopters. J. Intell. Robot. Syst. 1-31 (2020)

3. Martínez, J.R.G., Reséndiz, J.R., Prado, M.Á.M., Miguel, E.E.C.: Assessment of jerk performance s-curve and trapezoidal velocity profiles. In: 2017 XIII International Engineering Congress (CONIIN), pp. 1-7. IEEE (2017)

Publisher's Note Springer Nature remains neutral with regard to jurisdictional claims in published maps and institutional affiliations. 